\title{
'Five patients at the early stage of the Wuhan seafood market pneumonia virus outbreak' - bacterial load is much higher than SARS-Cov2 load, Pseudomonas is a definite problem in one patient, and Prevotella is abundant in all patients
}

Sandeep Chakraborty

\section{Letter}

Here, I analyze the metagenome from the bronchoalveolar lavage fluid of 5 Covid19 [1-3] patients from Wuhan (Accid:PRJNA605983, Table 1). There is no published study on this dataset yet.

\section{Very little viral load}

There is very little viral load (in the tens per million reads), similar to the metagenomic study in Brazil [4]. This is a very plausible cause for false negatives, as there is just not enough virus to detect. Thus, it is important to have a large set of RT-PCR primers to cover more of the genome.

\section{Much more bacterial load}

While there are a wide range of bacteria, Pseudomonas has definitely colonized patient1, while patient5 seems to have have the least bacterial load. Pseudomonas is associated with several diseases, several of which (pneumonia and septicemia) are relevant in Covid19 [5]. The bacterial species and their sequences are submitted in SI5patients on a per patient basis. These bacterial co-infections form the basis of hydroxychloroquine [6] and azithromycin working in clinical trials [7]. 
Table 1: Bacterial load is much higher than SARS-Cov2 load in five patients at the early stage of the Wuhan seafood market pneumonia virus outbreak: $\mathrm{PM}=$ per million. Number of reads ananlyzed: patient $1=11841722$, pat $2=12882084$, pat $3=12041947$, pat4 $=13333138$, pat5 $=23201546$. Pseudomonas is a definite secondary infection in patient1, while patient5 seems to have the least bacterial load. Patient 4 has the most viral load, otherwise viral load is quite low, just like in the patient from Brazil [4], explaining the high false negatives. As in all previous studies, Prevotella seems to be a common and abundant presence in all patients.

\begin{tabular}{|c|c|c|c|c|c|c|c|c|c|}
\hline patientient1 & $\mathrm{PM} /$ total & Patient2 & $\mathrm{PM} /$ total & Patient3 & PM/total & Patient4 & $\mathrm{PM} /$ total & Patient5 & PM/total \\
\hline Pseudomonas & $1529 / 18047$ & Prevotella & $125 / 1617$ & Sphingomonas & $351 / 4213$ & Capnocytophaga & $25 / 333$ & Neisseria & $11 / 260$ \\
\hline Sphingomonas & $1166 / 13766$ & Pseudomonas & $61 / 790$ & Prevotella & $157 / 1892$ & Veillonella & $18 / 250$ & Capnocytophaga & $4 / 113$ \\
\hline Clostridium & $400 / 4720$ & Streptococcus & $30 / 390$ & Pseudomonas & $141 / 1696$ & Streptococcus & $5 / 79$ & AlloPrevotella & $1 / 43$ \\
\hline Sphingobacterium & $326 / 3851$ & Clostridium & $28 / 370$ & Clostridium & $47 / 564$ & Cardiobacterium & $5 / 72$ & Prevotella & $1 / 38$ \\
\hline Lactobacillus & $244 / 2885$ & Haemophilus & $18 / 236$ & Escherichia & $36 / 440$ & Prevotella & $3 / 51$ & Haemophilus & $1 / 32$ \\
\hline Anaerocolumna & $217 / 2564$ & Lactobacillus & $14 / 181$ & Sphingobacterium & $32 / 386$ & Neisseria & $3 / 44$ & Coxiella & $1 / 26$ \\
\hline Delftia & $176 / 2084$ & Actinobacillus & $12 / 155$ & Streptococcus & $31 / 376$ & Schaalia & $2 / 33$ & Veillonella & $0 / 20$ \\
\hline Paenibacillus & $170 / 2006$ & Anaerocolumna & $10 / 133$ & Neochlamydia & $27 / 334$ & Ottowia & $1 / 14$ & Streptococcus & $0 / 17$ \\
\hline Neochlamydia & $168 / 1987$ & Escherichia & $10 / 129$ & Lactobacillus & $21 / 262$ & Coxiella & $0 / 13$ & Nocardioides & $0 / 16$ \\
\hline Methylorubrum & $150 / 1772$ & Neisseria & $9 / 118$ & Delftia & $19 / 232$ & Lautropia & $0 / 11$ & Campylobacter & $0 / 13$ \\
\hline Lysinibacillus & $148 / 1750$ & Delftia & $7 / 101$ & Methylorubrum & $17 / 206$ & Campylobacter & $0 / 10$ & Fusobacterium & $0 / 12$ \\
\hline Pelomonas & $123 / 1461$ & Paenibacillus & $7 / 99$ & Haemophilus & $17 / 204$ & Brachymonas & $0 / 8$ & Paracoccus & $0 / 12$ \\
\hline Prevotella & $106 / 1256$ & Sphingomonas & $7 / 99$ & Anaerocolumna & $16 / 199$ & Pseudomonas & $0 / 8$ & Pseudomonas & $0 / 11$ \\
\hline Chryseobacterium & $97 / 1155$ & Lysinibacillus & $6 / 78$ & Paenibacillus & $12 / 151$ & Eikenella & $0 / 7$ & Cardiobacterium & $0 / 10$ \\
\hline SARS-Cov2 & $78 / 923$ & SARS-Cov2 & $30 / 396$ & SARS-Cov2 & $18 / 222$ & SARS-Cov2 & $362 / 4821$ & SARS-Cov2 & $19 / 454$ \\
\hline
\end{tabular}


This study reenforces the role of Prevotella, which had taken over in a Chinese patient [8]. Prevotella also known to lower lymphocyte counts [9], increase IL-6 in plasma [10-12], cause ground glass opacity in lungs [13], and associated with cardiac injury [14] - all symptoms associated with Covid19. Here, a closely related species Alloprevotella is also abundant in one patient [15]. In 2003 SARS-Cov1 outbreak, secondary infection and IL-6 was highly correlated with hospitalization and deaths [16].

\section{References}

1. Perlman S (2020). Another decade, another coronavirus.

2. Wu F, Zhao S, Yu B, Chen Y, Wang W, et al. (2020) Complete genome characterisation of a novel coronavirus associated with severe human respiratory disease in Wuhan. China bioRxiv 24.

3. Chen L, Liu W, Zhang Q, Xu K, Ye G, et al. (2020) RNA based mNGS approach identifies a novel human coronavirus from two individual pneumonia cases in 2019 Wuhan outbreak. Emerging Microbes \& Infections 9: 313-319.

4. Chakraborty S (2020). Metagenome of sars-cov2 from a patient in brazil shows a wide range of bacterial species - lautropia, prevotella, haemophilus - overshadowing viral reads, which does not even add up to a full genome, explaining false negatives. doi:10.31219/osf.io/2xt3w. URL osf.io/2xt3w.

5. Bodey GP, Bolivar R, Fainstein V, Jadeja L (1983) Infections caused by pseudomonas aeruginosa. Reviews of infectious diseases 5: 279-313.

6. Schrezenmeier E, Dörner T (2020) Mechanisms of action of hydroxychloroquine and chloroquine: implications for rheumatology. Nature Reviews Rheumatology : 1-12.

7. Gautret P, Lagier JC, Parola P, Meddeb L, Mailhe M, et al. (2020) Hydroxychloroquine and azithromycin as a treatment of COVID-19: results of an open-label non-randomized clinical trial. International Journal of Antimicrobial Agents : 105949.

8. Chakraborty S (2020). The 2019 Wuhan outbreak could be caused by the bacteria Prevotella, which is aided by the coronavirus, possibly to adhere to epithelial cells - prevotella is present in huge aemounts in patients from both China and Hong Kong. doi:10.31219/osf.io/usztn. URL osf .io/usztn.

9. Shenker B, Vitale L, Slots J (1991) Immunosuppressive effects of prevotella intermedia on in vitro human lymphocyte activation. Infection and immunity 59: 4583-4589.

10. Leite AZ, Rodrigues NdC, Gonzaga MI, Paiolo JCC, de Souza CA, et al. (2017) Detection of increased plasma interleukin-6 levels and prevalence of Prevotella copri and Bacteroides vulgatus in the feces of type 2 diabetes patients. Frontiers in immunology 8: 1107.

11. Larsen JM (2017) The immune response to Prevotella bacteria in chronic inflammatory disease. Immunology 151: 363-374.

12. Choi EY, Jin JY, Choi JI, Choi IS, Kim SJ (2014) Effect of azithromycin on Prevotella intermedia lipopolysaccharide-induced production of interleukin-6 in murine macrophages. European journal of pharmacology 729: 10-16.

13. Berardino ADM, Inchingolo R, Smargiassi A, Re A, Torelli R, et al. (2014) Empyema caused by prevotella bivia complicating an unusual case of spontaneous chylothorax. Journal of clinical microbiology 52: $1284-1286$. 
14. Dorn BR, Dunn WA, Progulske-Fox A (1999) Invasion of human coronary artery cells by periodontal pathogens. Infection and immunity 67: 5792-5798.

15. Downes J, Dewhirst FE, Tanner AC, Wade WG (2013) Description of Alloprevotella rava gen. nov., sp. nov., isolated from the human oral cavity, and reclassification of Prevotella tannerae moore et al. 1994 as alloprevotella tannerae gen. nov., comb. nov. International journal of systematic and evolutionary microbiology 63: 1214 .

16. Jiang Y, Xu J, Zhou C, Wu Z, Zhong S, et al. (2005) Characterization of cytokine/chemokine profiles of severe acute respiratory syndrome. American journal of respiratory and critical care medicine 171: $850-857$. 